TM-1476

[SSC-N-386]

\title{
Cryogenic Instrumentation of an SSC Magnet Test Stand*
}

K. McGuire, J. Strait, M. Kuchnir, and A. McInturff

Fermi National Accelerator Laboratory

P.O. Box 500, Batavia, Illinois 60510

September 1987

*Presented at the 1987 Cryogenic Engineering Conference/Intermational Cryogenic Materials Conference, St. Charles, Illinois, June 14-18, 1987. To be published in the proceedings Advances in Cryogenic Engineering. 


\title{
CRYOGENIC INSTRUMENTATION OF AN SSC MAGNET TEST STAND
}

\author{
K. McGuire, J. Strait, M. Kuchnir, and A. McInturff \\ Fermi National Accelerator Laboratory \\ Batavia, Dllinois
}

\begin{abstract}
This paper describes the system used to acquire cryogenic data for the testing of SSC magnets at the Fermilab Magnet Test Facility. An array of pressure transducers, resistance thermometers, vapor pressure thermometers, and signal conditioning circuits are used. Readings with time resolution appropriate for quench recording are obtained with a waveform digitizer and stesdy-state measurements are obtained with higher accuracy using a digital voltmeter. The waveform digitizer is clocked at a $400 \mathrm{~Hz}$ sampling rate and these readings are stored in local ring buffers. The system is modular and can be expanded to add more channels. The software for the acquisition, control, logging, and display of cryogenic data consist of two programs which run as separate tasks. These programs (as well as a third program which acquires quench and magnetic data) communicate and pass data using shared global resources. The acquired data are available for analysis via a nationwide DECnet ${ }^{\circ}$ network.
\end{abstract}

\section{INTRODUCTION}

Fermilab is collaborating witb Brookhaven National Laboratory, Lawrence Berkeley Laboratory, and the SSC Central Design Group on developing, building, and testing superconducting magnets ${ }^{1,2}$ for the proposed Superconducting Supercollider (SSC) ${ }^{3}$. Quench and magnetic field tests ${ }^{4,6}$ on these magnets are performed by the Fermilab Magnet Test Facility. Temperature and pressure measurements are necessary to characterize the thermodynamic state of the helium coolant. The temperature of the magnet coil must be known to an accuracy better than $50 \mathrm{mK}$ to understand both the quench performance and the magnetic field quality dats. Temperatures and pressures are measured dynamically during quenches to locate the quench origin longitudinally and to understand stresses on the magnet and cryostat.

Figure 1 shows a flow schematic of the test stand and the locations of the sensing devices. Liquid helium enters the supply end box (feed can) in the 'Liquid Supply' line, where some of the flow is diverted to cool the power leads. The inlet temperature is then measured by a carbon resistor before the fluid enters a subcooler. The liquid level in this subcooler is measured both by a differential preasure gauge ${ }^{b}$ and by a superconducting wire probe ${ }^{c}$. The temperature of the subcooled liquid is measured with a helium vapor pressure thermometer ${ }^{d}$ (VPT) and its pressure is measured by a warm pressure transducer ${ }^{e}$ whose tap is after the ubcooler. In both magnet interconnect regions, where the transition is mode between the end boxes and the magnet, there are a germanium', a carbon glaso, and a platinum ${ }^{h}$ resistor for measuring the temperature, and a cold pressure transduceri. At the return end the 
helium is directed back to the supply end box through the 'Liquid Return' line of the magnet cryostat. It then enters the supply end box where its temperature is measured by a VPT, the pressure is measured by a warm pressure transducer, and the mass flow is measured by a venturi tube. The venturi tube was installed at this point due to space requirements inside the feed can. Some of the returning liquid is used to maintain the liquid level in the subcooler. The remaining helium passes through a Joule-Thomson valve and enters the magnet's 'Gas Return' line. Pressure is measured at this point by an inexpensive piezoresistive bending beam pressure transducer' and the temperature is measured by a carbon resistork. The fluid then passes through the magnet and is again turned around to pass through the magnet' $\mathrm{B}$ ' $20 \mathrm{~K}$ Shield' before returning to the refrigerator. In this mode of cooling, this shield actually operates at about $4.5 \mathrm{~K}$. The instrumentation in the feed can for the ' $20 \mathrm{~K}$ Shield' is the same as that used for the 'Gas Return' line. Instrumentation for the ' $80 \mathrm{~K}$ Shield', which is cooled by liquid nitrogen, consists of platinum resistors at either end, as well as a pressure gauge at the feed end and a flowmeter at the return end, where the nitrogen is warmed up by a heat exchanger and exhausted.

\section{SENSING DEVICES}

Pressure measurements were obtained using several different types of pressure transducers. In the locations where high accuracy was required, bonded strain gauge $e^{t}$ transducers were used. Cold pressure transducers of the sputtered strain gauge ${ }^{i}$ type were installed inside the magnet interconnect region to obtain readings witb high time resolution. Although these transducers have large zero shift between 4 and $300 \mathrm{~K}$, their sensitivity is unchanged. They have an excellent time response because they are located directly in the fluid, with no intervening tubing. Delays introduced by the tubing connecting the warm pressure transducers measure about $30 \mathrm{~ms}$ at the feed end and $15 \mathrm{~ms}$ at the return end of the magnet. Using the cold pressure transducers, the leading edge of pressure pulses caused by quencbing can

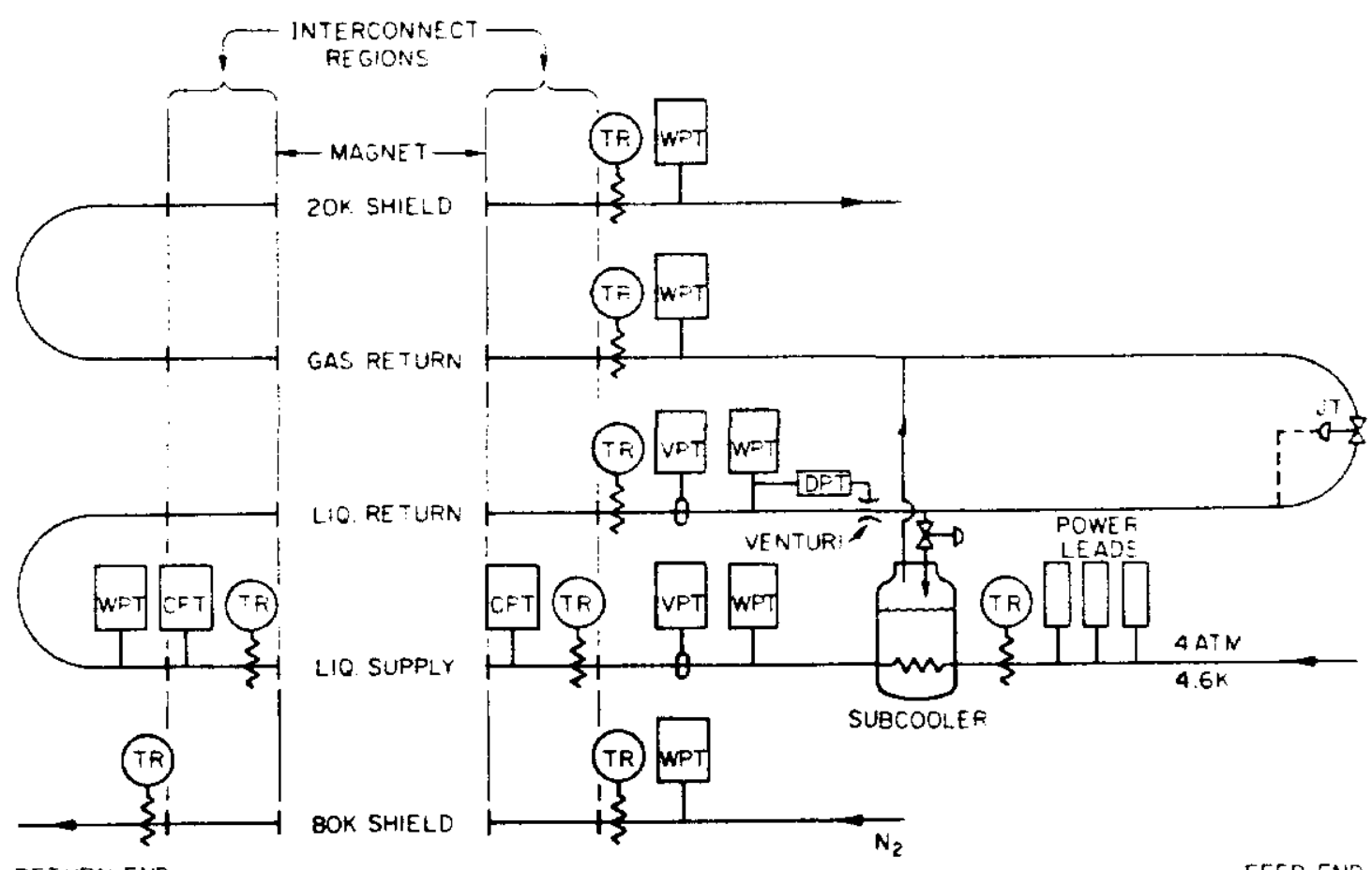

RETURN END

LEGEND:

CPT - COLO PRE SSURE TRANSOUCER WPT- WARM PRESSURE TRANSOUCER VPT - VAPOR PRESSURE THERMOME TER
TR - RESISTANCE THERMOMETERS

DPT - DIFFERENTIAL PRESSURE TRANSDUCER

Fig. 1. Simplified flow echematic thowing locations of instrumentation. 
be observed with resolution of about $2 \mathrm{~ms}$, corresponding to a resolution of $0.3 \mathrm{~m}$ in the longitudinal origin of the quench. The signals from the pressure transducers are amplifed at the test atand before being cabled into the control room.

Mass flow of helium through the magnet is measured with a venturi tube and differential pressure tranaducer combination. Turbine flowmeters were ruled out due to planned quencb testing of the magnets. Fast pressure rises, such as those expected when the magnet quenches would destroy these devices.

Several different types of resistance thermometers were used for various reasons having to do with range, accuracy, repeatability, and time response. These thermometers, as well as their ranges and reasons for usage are shown in Table 1 . In the locations where high accuracy is required, a germanium resistor and a carbon glass resistor are paired. There are two reasons for this: the first reason is redundancy; the other has to do with the accuracy and the effects of magnetic fields on these devices. An attempt is made to combine the best features of these thermometers and to compensate for their weaknesses. The germanium resistors are exceptional in their sensitivity, repeatablity, and time response, but are susceptible to the effects of magnetic fields. The magnitude of this effect is orientation-dependent. While a prototype of the system was being tested, it was possible to sermal oscillation with a frequency of $20 \mathrm{~Hz}$ with a peak-peak amplitude corresponding to $1 \mathrm{mK}$ (excitation was 10 microamperes). The frequency of this oscillation was made to change by altering the cryogenic configuration of the test stand in which this thermometer was installed. The carbon glase thermometer has good accuracy (approximately as accurate as a silicon diode 8 t $4.2 \mathrm{~K}$ ) and is not very susceptible to magnetic fields. The carbon glass thermometer can be crosscalibrated against the germanium thermometer in the absence of magnetic fields. Then the carbon glass thermometer can be used as the primary thermometer when there are magnetic fields present.

Some of the resistance thermometers were initially calibrated at the factory from which they were purchased. All of them were mounted, packaged, and calibrated, 6,0 at the Fermilab Magnet Development Facility. After about a year of service (and fve thermal cycles), some germanium and carbon glass thermometers were recalibrated. The new calibration was found to agree with the original calibrations (including independent factory calibration) to within 5 $\mathrm{mK}$.

The resistance thermometers are excited by a DC constant-current source. Depending on the type of thermometer used, the excitation current is 25,6 , or 1.5 microsmperes. The current for all thermometers on a single current source runs in series through a resistor at

Table 1. Characteristics of Resistance Thermometers

\begin{tabular}{|c|c|c|c|}
\hline Thermometer & $\begin{array}{c}\text { Range } \\
\text { (kelving) }\end{array}$ & Recsons for use & Disadvantages \\
\hline Germanium & $1-80$ & $\begin{array}{l}\text { Excellent stability - repeatability is } \\
\pm 0.5 \mathrm{mK} \text { when thermally shocked } \\
\text { between } 295 \mathrm{~K} \text { and } 4.2 \mathrm{~K} \\
\text { Excellent time response }\end{array}$ & $\begin{array}{l}\text { Influenced by } \\
\text { magnetic fields } \\
\text { Small range }\end{array}$ \\
\hline Carbon glass & $2-200$ & $\begin{array}{l}\text { Good stability - repeatability is } \\
\pm 7.5 \mathrm{mK} \text { when thermally shocked } \\
\text { between } 295 \mathrm{~K} \text { and } 4.2 \mathrm{~K} \\
\text { High sensitivity below } 10 \mathrm{~K} \\
\text { Good immunity to magnetic fields }\end{array}$ & \\
\hline Carbon & $2 \cdot 200$ & Low cost & $\begin{array}{l}\text { Unstable with } \\
\text { thermal cycling }\end{array}$ \\
\hline Platioum & $25-800$ & Used to monitor cooldown and warmup & \\
\hline
\end{tabular}


room temperature (designated $R_{I}$ ) and then through the thermometers. The resistor at room temperature, $R_{l}$, has voltage taps on it. By knowing the value of $R_{I}$ and the voltage drop across it, the current through any thermometer in the chain is known. Four leads (made of twisted pairs of manganin wire) from each thermometer are brought outside the cryostat and the connections to complete the current chain are made externally. This allows thermometers to be bypassed in the event that there is a short circuit or they become open-circuited. It also allows the electronics to be modular. Provisions for changing the direction of the current to measure the offsets due to thermal EMFs and contact potentials is provided by mechanical relay. This relay can be controlled either locally by a switch on the front panel, or remotely by applying a TTL level signal to a jack on the front panel. To minimize noise pickup, The voltage drops across these thermometers are first amplified locally (see Fig. 2) at the test stand before being cabled into the control room (where they are measured by the readout equipment). These amplifiers have a low impedance single-ended output and the readout devices have high impedance differential inputs.

While the accuracy of the resistance thermometry measurements has not been studied in detail, it is believed that the dominant source of uncertainty comes from the stability of the amplifiers. These problems can be minimized by rebuilding with higher grade components. An error in the gain of $1 \%$, due to thermal or temporal drifts over the approximately 1 month testing time of each magnet, corresponds to an error of about $20 \mathrm{mK}$ for the germanium and carbon-glass thermometers. The internal consistency of the measurements suggest that this is a reasonable estimate of the accuracy. Uncertainties from other sources, in particular the calibration, are considerably smaller than this.

Helium vapor pressure thermometers (VPTs) are used in two locations to check the calibrations of some of the resistance thermometers. The geometry of the VPTs are dictated by the absolute accuracy required and the warm volume of the system (tubing and pressure transducers). Because the absolute accuracy of strain gauge pressure transducers is specified as a percentage of the full scale range, it is desirable to keep this range as amall as possible. Since the maximum pressure for helium vapor in equilibrium with liquid is about $227 \mathrm{kPa}$, the volumes of the system were chosen such that the charging (warm) pressure of the system is alightly higher than this. A large cold volume was used for two reasons - to yield a large dynamic range, and so that errors introduced by uncertainty in the warm volumes would be as small as possible. The volume of the bulb is $0.003 \mathrm{~L}$, while the volume of the cold internal tubing is $0.009 \mathrm{~L}$. The warm volume, consisting of tubing and cavities within pressure gauges and transducers is $0.971 \mathrm{~L}$, including $0.906 \mathrm{~L}$ ballast volume. The final charging pressure is $265 \mathrm{kPa}$. The calculated time constant is about 0.17 seconds and the operating range (corresponding to the bulb being $10 \%$ full to $90 \%$ full) is from $2.2 \mathrm{~K}$ to $5.1 \mathrm{~K}$. The primary error in

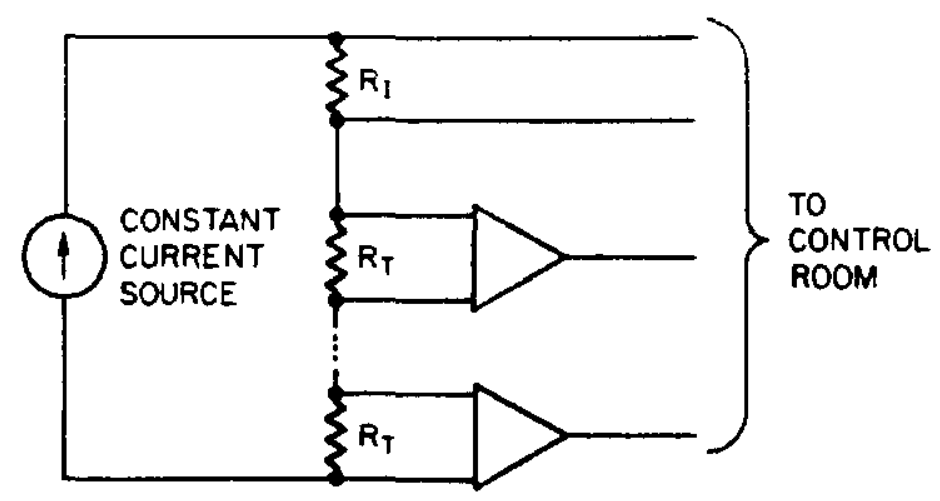

Fig. 2. Implementation of resistence thermometers. 
reading the VPT. comes from the accuracy in the pressure transducers ${ }^{m}$ used to read them and their associated electronics $(0.05 \%$ of Full Scale Output). Using a pressure transducer with a range of 50 psia $(340 \mathrm{kPa})$, the accuracy obtained is better than $2 \mathrm{mK}$.

Table 2 summarizes the accuracy of the measurements.

\section{READOUT DEVICES}

The required measurements fall into two categories - those which must be done very fast (where absolute accuracy is not important), and those which must be done with high accuracy (where speed is not required). Since no readout device has the capability of high accuracy and great speed, it was decided to use two readout devices. The fast resdings are taken with a multichannel waveform digitizer ${ }^{n}$ with 12-bit resolution (10-bit accuracy). The memory in these modules is implemented as sets of ring buffers for each channel. As the waveform digitizer is clocked, a pointer to the next memory location is incremented. When the pointer reaches the end of the ring buffer, it is reset to point to the beginning. This cycle is repeated continuously until a 'stop trigger' is generated when a quench is detected. The waveform digitizer is configured (by a combination of jumpers and software) such that it take s certain number of post-trigger samples when the stop trigger is received. By judiciously choosing the number of post-trigger samples, a number of pre-trigger samples can be saved as well.

A digital voltmeter ${ }^{\circ}$ (DVM) is used for the high accuracy readings. Since most DVMs have at most two inputs, it is impractical to use several of these when there are large numbers of devices to read. Therefore, the signals are multiplexed through a scanner ${ }^{p}$. The scanner uses mechanical relays for switching so as not to degrade or corrupt the signal, and sequentially switches the signal to the DVM.

Table 2. Accuracy of Measurements.

\begin{tabular}{|l|l|l|l|}
\hline \multicolumn{1}{|c|}{ Location } & Measurement & \multicolumn{1}{|c|}{ Device Type } & \multicolumn{1}{|c|}{ Accuracyn } \\
\hline \hline Power leads & Temperature & Carbon resistor & $50 \mathrm{mK}$ \\
\hline Magnet flow & Flow & Venturi / strain gauge & unknown \\
\hline Liquid supply & Pressure & Bonded strain gauge & $0.375 \mathrm{psi}(2.6 \mathrm{kPa})$ \\
\hline Liquid supply & Pressure & Sputtered strain gauge & $0.625 \mathrm{psi}(4.3 \mathrm{kPa})$ \\
\hline Liquid supply & Temperature & Germanium resistor & $20 \mathrm{mK}$ \\
\hline Liquid supply & Temperature & Carbon glass resistor & $20 \mathrm{mK}$ \\
\hline Liquid supply & Temperature & Carbon resistor & $50 \mathrm{mK}$ \\
\hline Liquid supply & Temperature & VPT / bonded strain gauge & $2 \mathrm{mK}$ \\
\hline Liquid return & Pressure & Bonded strain gauge & $0.375 \mathrm{psi}(2.6 \mathrm{kPa})$ \\
\hline Liquid return & Pressure & Sputtered strain gauge & $0.625 \mathrm{psi}(4.3 \mathrm{kPa})$ \\
\hline Liquid return & Temperature & Germanium resistor & $20 \mathrm{mK}$ \\
\hline Liquid return & Temperature & Carbon glass resistor & $20 \mathrm{mK}$ \\
\hline Liquid return & Temperature & Carbon resistor & $50 \mathrm{mK}$ \\
\hline Liquid return & Temperature & VPT / bonded strain gauge & $2 \mathrm{mK}$ \\
\hline Gas return & Pressure & Piezo-resistive on bending beam & $0.25 \mathrm{psi}(1.7 \mathrm{kPa})$ \\
\hline Gas return & Temperature & Carbon resistor & $50 \mathrm{mK}$ \\
\hline 20K shield & Pressure & Piezo-resistive on bending beam & $0.25 \mathrm{psi}(1.7 \mathrm{kPa})$ \\
\hline 20 K shield & Temperature & Carbon resistor & $50 \mathrm{mK}$ \\
\hline 80 K shield & Pressure & Piezo-resistive on bending beam & $0.25 \mathrm{psi}(1.7 \mathrm{kPa})$ \\
\hline 80 K shield & Temperature & Carbon resistor & $50 \mathrm{mK}$ \\
\hline
\end{tabular}

- Accuracy quoted for thermometers are opecified at $4.2 \mathrm{~K}$.

Accuracy quoted for preasure traneducers are from mannfucturer's opecifications. 
The base of the readout system consists of a CAMAC ${ }^{\circ}$ crate connected to a VAX' computer through a CAMAC interfacer. Both the DVM and the scanner are controlled by the computer through \& GPIB ${ }^{10,11,4}$ interface which resides in the CAMAC crate. Figure 3 shows a composite of the test stand instrumentation and the associsted resdout equipment.

\section{OPERATOR INTERFACE}

Measurements of pressure and flow are provided to the cryogenic systems operator in two different ways. First there is a gauge panel on the test stand with various pressure gauges and flowmeters which can be read visually. Temperatures at two locations can also be read visually using pressure gauges on the VPTs. All instrumented readings can be obtained from second source. A cryogenic data acquisition program functions as a data server. It receives data requests from a separate data logging program which is controlled by timers implemented in software. There are separate timers for each of three data logging functions: logging data to a computer file, logging dats to a printer, and updating a data display on a computer terminal. This display functions as the operator's primary interface for high accuracy data. When a timer times out, it specifies the appropriate data request by setting bits in a common (global) event flag cluster. It then notifies the cryogenic data acquisition program that it wants data by writing a message to a shared 'mailbox'. The requested data is then acquired and then stored in shared global memory. Completion of the transaction is signalled by a 'Data Ready' event flag. Finally, the data logging program collects the data from the shared global memory and performs the appropriate logging function. Cryogenic dats for the program which does the quench and magnetic measurements is obtained the same way.

\section{DATA SHARING}

Since the SSC project is a collaboration between Fermilab, Brookhaven National Laboratory, Lawrence Berkeley Laboratory, and the SSC Central Design Group, it is useful to have the data acquired during measurements available on-line to all parties involved. This is easily accomplished with a nationwide network of VAX computers which use DECnet software for

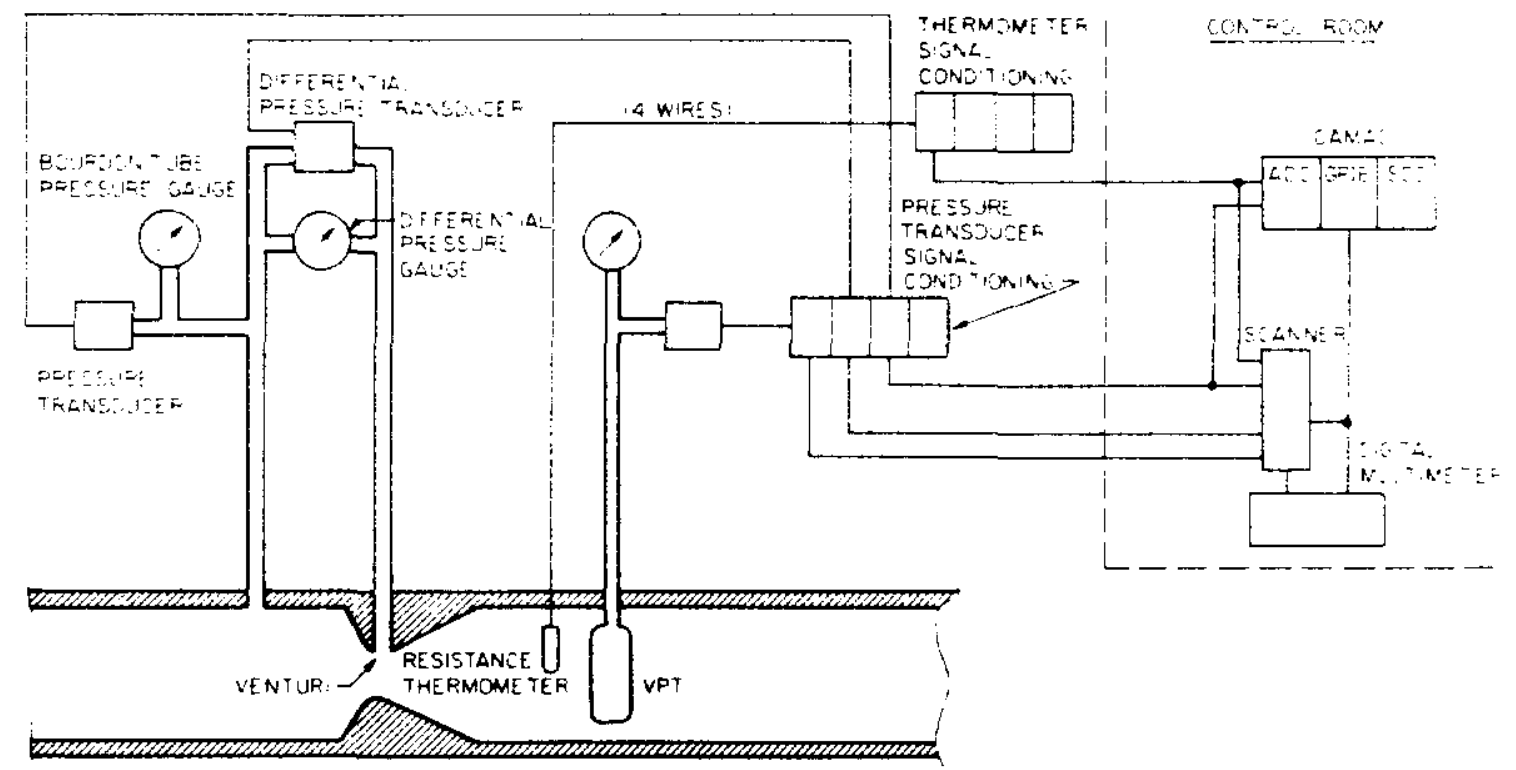

Fig. 3. Composite of test stand instrumentation. 
transparent file transfers. Dats are initially stored on the VAX at the Fermilab Magnet Test Facility. From there, files can be copied to any computer on the network for special studies and analysis, and are instantly available as the measurements proceed.

\section{ACKNOWLEGEMENTS}

Fermilab is operated by the Universities Research Associstion under contract with the United States Department of Energy. Many people have contributed to the success of this project by providing the necessary support. In particular, we wish to acknowlege the contributions of R. Barger, A. Bianchi, B.C. Brown, J. Garvey, F. Johnson, G. Kirschbaum, D. Lewis, R. Nehring, J. Pachnik, A. Rusy, E. Schmidt, H. Stahl, J. Tague, F. Wilson, and W. Zimmerman. In addition, we wish to acknowlege the work of the Fermilab Accelerator Division Cryogenic Systems group, who designed and built the end boxes, particularly T. Peterson and J. Theilacker; and J. Osterlund and J. Sondericker of Brookhaven National Laboratory, who were consulted in the design stage of the resistance thermometry.

\section{MANUFACTURERS}

a. DECnet is a trademark of Digital Equipment Corporation, Massachusetts.

b. Model Capsuhelic, Dwyer Instruments, Michigan City, Indiana.

c. American Magnetics, Oak Ridge, Tennessee.

d. Designed by M. Kuchnir, Fermilab.

e. Model 831 Dynisco, Norwood, Massachusetts.

f. Model N2K, Scientific Instruments, Inc., West Palm Beach, Florida.

g. Model CGR-1-1000, LakeShore Cryotronics, Inc., Westerville, Ohio.

h. Model P1, Scientific Instruments, Inc., West Palm Beach, Florida.

i. Model 1000-05, I.M.O. Delaval, CEC Instruments Division, Pasadena, California.

j. Model PX105, Omega Engineering, Inc., Stamford, Connecticut.

k. Ordinary carbon resistors designed for electronics use, Allen-Bradley, lnc.

1. Model TJE Differential Pressure Transducer, Sensotec, Columbus, Ohio.

m. Model Super TJE Absolute Pressure Transducer, Sensotec, Columbus, Ohio.

n. LeCroy Model 8212A High Accuracy Simultaneous Sampling Data Logger, LeCroy Research Systems Corporation, Spring Valley, New York.

o. Model 3457, Hewlett-Packard, Palo Alto, California.

p. Model 705, Keithley Instruments, Inc., Cleveland, Ohio.

q. Model 11/730, Digital Equipment Corporation, Massachusetts. VAX is a trademark of Digital Equipment Corporation.

r. Model 4I1 PDP-11 CAMAC Interface, Jorway Corporation, Westbury, New York.

8. Model 3388-GIA GPIB Interface Module, Kinetic Systems Corporation, Lockport, Dlinois.

\section{REFERENCES}

1. P. Dahl, et al., Construction of cold mass assembly for full length dipoles for the SSC accelerator, "Proceedings of the Applied Superconductivity Conference," Baltimore, Maryland (1986), J. Schooley (Ed.).

2. R.C. Niemann, et al., Design, construction and test of a full scale SSC dipole magnet cryostat thermal model, "Proceedings of the Applied Superconductivity Conference," Baltimore, Maryland (1986), J. Schooley (Ed.).

3. Conceptual Design of the Superconducting Super Collider, SSC Central Design Group, Lawrence Berkeley Laboratory, One Cyclotron Road, Berkeley, California, 94720. 
1. J. Strait, et al, Full length prototype SSC dipole test results, "Proceedings of the Applied Superconductivity Conference," Baltimore, Maryland (1986), J. Schooley (Ed.). Also available as Fermilab Technical Memo TM-1450, SSC-N-320, 0102.001 .

5. J. Strait, et al., Teats of prototype SSC magnets, "Proceedings of the 12th Particle Accelerator Conference," Washington, D.C. (1987). Also available as Fermilab Technical Memo TM-1451, SSC-N-321, 0102.001.

6. M. Kuchnir, J.L. Tague, Carbon Resistance Thermometers, Fermilab Technica] Merno TM-647, 1600.000.

7. M. Kuchnir, J.L. Tague, and H. Cranor, Pulsed Current Resistance Thermometry, Fermilab Technical Memo TM-1099, 1680.00.

8. M. Kuchnir, Pulsed current resistance thermometry, in "Advances in Cryogenic Engineering" Vol. 29, Plenum, New York, (1984).

9. CAMAC stands for Computer Automated Messurement and Control, and is specified by IEEE standards $583,595,596,675$, and 683 .

10. GPIB stands for General Purpose Interface Bus, and is also known as HPIB (Hewlett-Packard Interface Bus) and ASCII bus. It is specified by IEEE Std. 488 and ANSl Std. MC1.1.

11. Kevin McGuire, Fermilab Magnet Development and Test Facility GPIB Package, Fermilab Technical Memo TM-1287, 2320.000. 